\title{
Participatory community singing program to enhance quality of life and social and emotional well-being in Aboriginal and Torres Strait Islander Australians with chronic diseases
}

\begin{abstract}
Objective: The objective of this study was to assess the effectiveness of a 12-month community singing program on quality of life (QoL) using survey methods.

Methods: We prospectively collected data on Aboriginal and Torres-Strait Islander people with chronic diseases involved in a participatory community singing program. Aboriginal and Torres Strait Islander people with a chronic disease and an average age of 51 years (range, 18-85 years) self-selected to participate in the singing group $(n=45)$, which included a weekly singing rehearsal and a monthly performance. There were 27 people in the control group. We compared the intervention and control groups by evaluating the QoL, social and emotional well-being, health behaviors, social isolation, and loneliness at a baseline and at 12 months.

Results: At 12 months, those who participated in the singing program showed a significant increase in QoL, reduced stress, and a significant reduction in social isola. tion and loneliness compared with patients in the control group.

Conclusions: The community singing program may enhance social and emotional well-being and QoL in Aboriginal and Torres Strait Islander people with chronic diseases, and the singing program is a viable health promotion intervention program for this population.
\end{abstract}

Keywords: Aboriginal and Torres Strait Islander Australians; chronic diseases; participatory community singing program; quality of life; social and emotional well-being.

\footnotetext{
*Corresponding author: Jing Sun, School of Public Health and Griffith Health institute, Griffith University, Gold coast Campus, Parkland, Southport, Queensland 4222, Australia, Phone:+61·7·55527875, E.mail: j.sun@griffith.edu.au Nicholas Buys: School of Human Services and Social Work and Griffith Health Institute, Griflith University, Southport, Queensland, Australia
}

\section{Introduction}

Poor quality of life (QoL) is a common feature of people with multiple chronic diseases. Chronic diseases, including coronary heart disease, heart failure, and their concomitant symptoms including hypertension, diabetes, and severe depression, are associated with reduced health-related QoL $[1,2]$. The health status of Aboriginal and Torres Strait Islander Australians exemplifies this situation. They not only have a higher prevalence of chronic disease than the normal population but their conditions are often caused by social and emotional difficulties $[3,4]$.

In populations with chronic disease, health-related QoL is an important outcome measure comprising the subjective appraisal of a person's mental, physical, and social functioning. These QoL-related risk factors may contribute to the development and recurrence of multiple chronic diseases $[1,2,5]$. Chronic conditions are often associated with a range of social and emotional factors, including poor levels of resilience, high levels of depression, inability to recover from stressful life events, social isolation, and loneliness. Addressing these QoL issues is therefore as important as the provision of medical treatment for the underlying chronic diseases.

A major goal of a chronic disease secondary prevention program is therefore to improve the patient's QoL [6] because self-reported QoL is an important predictor of mortality and hospitalizations due to recurrent chronic disease events $[7,8]$. Two studies $[1,5]$ found a positive relationship between psychological health and mental and physical health in patients with multiple chronic diseases, independent of covariates. These relationships were cross-sectionally observed [5] as well as 12 months after an intervention program [9]. Several studies report on the association between QoL and sociodemographic variables [5], chronic disease risk factors, and severity of disease [9]. However, there is a lack of knowledge concerning the impact of QoL on sacial and emotional wellbeing on Aboriginal and Torres Strait Islander people. It is 
therefore important to investigate this relationship and at the same time control for potential confounding variables such as social economic factors.

A participatory community singing program was chosen as an appropriate intervention to examine this relationship because it has been shown to be effective in promoting QoL in people with mental and physical health problems in other populations [10]. Group singing is an aerobic exercise. It is widely practiced in many countries and has gained increasing recognition in Australia [11, 12]. Recent literature has suggested that it may be particularly suitable for the patients with chronic disease [13]. Singing incorporates heart and lung muscle exercise and gentle physical activity with relaxation and deep breathing. Reported benefits of singing include reduced depression and anxiety [10] and improved self-efficacy and confidence [14]. Improved exercise capacity and QoL have not been reported in patients with multiple chronic diseases. Furthermore, it is culturally relevant for Aboriginal and Torres Strait Islander populations, in which singing is an important community-based activity [12].

We hypothesized that group singing may enhance QoL in patients with chronic disease and may be associated with an improvement of social and emotional well. being in Aboriginal and Torres Strait Islander people with chronic diseases. Our study therefore compared the differences in QoL preintervention and postintervention in this population and determined the relationship between QoL and depression, social and emotional well-being, resillence, social isolation, and loneliness.

\section{Methods}

A sample of 133 patients with more than two chronic disease conditions were recruited from five community-controlled health services (CCHSs) in South-East Queensland in Queensland, Australia. Chronic conditions included heart disease, diabetes, cancer, depression, and psychosis. Our research was participatory in nature, with local Aboriginal community leaders playing important central roles in the design and implementation of the study. Participants were re. cruited through Aboriginal CCHSs when they attended health check consultations. The individuals included in this investigation were 18 years or older, consented to participate in the intervention program, completed baseline assessment, intervention components, and postintervention assessment, and showed interest in singing activities. Patients who were not able to attend the intervention components or were not interested in the singing activities were asked to be in the control group. There were 62 patients in the baseline assessment time, and 45 of them stayed in the intervention group until the 12-month postintervention time. There were 71 patients in the control group, and 27 of them stayed until the postintervention assessment time. Patients who had major changes in their cardiac medical regimen in the previous 3 months, a major cardiac event or procedure in the previous 3 months, unstable arrhythmias or major valvular disease, severe-level stroke and psychiatric condition, or were currently participating in a conventional cardiac reha bilitation program were excluded from the study. Written informed consent was obtained from each participant before preintervention screening, and ethical approval was obtained through the Research Ethics Committee at the Griffith University of Australia (GU Ref No. $\mathrm{PBH} / 13 / 10 / \mathrm{HREC}$ ).

CCHSs conducted and coordinated the intervention programs, or ganizing intervention activities, weekly rehearsals, testing, and sing. ing performances. The intervention consisted of 2 h group singing classes held weekly for 12 months. Classes included warm-up exercises of the body, breathing techniques, and releasing tension, which are then followed by singing rehearsals. Patients were encouraged to practice at home. A more detailed description of the methods and intervention protocol may be found elsewhere [12].

\section{QoL and social and emotional well-being assessment}

Data collection occurred at baseline and 12 months, QoL was meas ured using the singing-related Qol, questionnatre. This instrument consists of 21 items covering physical, psychological, and positive affect [10] and quantifies the QoL, related to each item on a 3-point response scale. Scores on the scale range from 0 to 63 , with a higher score denoting a more favorable Qol, as a result of participation in singing related activities. The total variance explained by the 21 items for the singing-related QoL is $66.73 \%$, indicating excellent content validity for the QoL for the current study sample. The 21 items were divided into two subscales through factor analysis; singing-related QoL and positive affect. The reliability analysis demonstrated a high Cronbach $\alpha$ coefficient of 0.97 for singing-related QoL and 0.99 for positive affect. The overall reliability was 0.99 for the singing-related QoL questionnaire.

To measure stressful life events, participants answered 15 questions (in a yes/no format) that have been validated in Aboriginal and Torres Strait Islander people [15]. The questions covered al. cohol and drug use, gambling problems, jail-related experience, inability to find a job and job loss, abuse, discrimination, fighting, family member death, and overcrowding at home. High scores demonstrate a high stress level. Factor analysis was used to validate the 15 questions in participants with chronic disease patients. It revealed four subscales from the sample, including alcohol- and drug use-related stress (4 items), abuse and fighting-related stress ( 5 items), job loss and inability to find job ( 4 items), and family member death and crowding-related stress ( 5 items). The four sub. scales had reasonable level of reliability, with Cronbach $\alpha$ coeffi cients of $0.70,0.60,0.49$, and 0.45 , respectively. The overall reliability for the stress questions is acceptable, witl a Cronbach $\alpha$ coefficient of 0.71 .

To measure social and emotional well-being, patients answered six questions. This test has been previously used to assess and deter. mine the social and emotional difficulties level of the Aboriginal and Torres Strait people and has been validated on this population [16]. Questions related to social and emotional difficulties were asked on a 3-point Likert scale, with a high score indicating higher levels of social and emotional difficulties. 
Participants answered 20 questions related to social isolation and loneliness [17]. This questionnaire has been validated in a normal population and was used to determine the symptoms of social isolation and loneliness. The questions were asked on a 6-point Likert scale ranging from 'strongly disagree' to 'strongly agree'. Factor analysis revealed two subscales: social isolation, with 10 items reflecting feelings of disconnection with community and social reality, and loneliness, with eight items demonstrating feelings of loneliness and lack of social support. The other two items relating to friends' support and friendship relationship had low levels of reliability and were therefore deleted from the data analysis. The reliability for the social isolation and loneliness was high, with Cronbach $\alpha$ coefficients of 0.95 and 0.92 and an overall reliability level for the social isolation questionnaire of 0.92 . A high score on this scale indicates a high level of social isolation and loneliness.

\section{Statistical analysis}

The baseline characteristics of the study patients were compared using t-tests for continuous variables and Fisher's exact test for categorical variables. If the demographic variables or disease conditions were different between the intervention and the control groups, these variables will be considered as confounding factors and will be entered as the subsequent univariate analysis of variance (ANCOVA) model. The ANCOVA was tsed to compare the changes in QoL scores, stress, depression, subscales of stresses, and resilience after 12 months between the intervention and the control groups when confounding factors are controlled in the analysis.

Multiple linear regression analysis was used to determine the ef fect of singing-enhanced QoL on factors including life stresses, social and emotional well-being, social isolation, loneliness, and resilience, whereas confounding factors (e.g., age, gender, disease conditions) are controlled in each model. The multiple linear regression analy. sis was repeated for the analysis to lest the effect of singing-related relaxation on stresses, social and emotional well-being, social isola tion, Ioneliness, and resilience.

\section{Results}

\section{Subject characteristics}

Table 1 shows that the mean age of study patients was 51.60 years for the singing group and 51.48 years for the control group. Women comprised the majority of the participants in both the singing and the control groups. Participants in both groups were on various medications, with the length of medication use of participants in the intervention group being 112 months and 129 months in the control group. There were no statistically significant differences between the groups with regard to demographics, rates of cardiovascular-related disease, length of medication use, and other comorbidities except for diabetes and asthma. More people in the control group had diabetes and asthma coupled with heart disease.

\section{Singing-related QoL}

Table 2 presents the change in mean outcome measures over the $12 \cdot$ month period. When the singing group was compared with usual care alone, statistically significant improvements were seen, with increases in singing-related mental and physical health aspects of QoL and positive affect ( $p<0.001$ and $p<0.001$, respectively). Table 2 also presents the change in other measures over 12 months. In the singing group, there are overall reductions in stressful events, depression level, sense of social isolation, and loneliness, as compared with the control group, although this difference did not reach statistical significance.

There were significant differences between the singing and the control groups in the reduction of alcohol and

Table 1 Baseline characteristics of the study population.

\begin{tabular}{|c|c|c|c|c|}
\hline Characteristics & Singing $(n=45)$ & Control $(n=27)$ & $\operatorname{tor} \chi^{2}$ & p.Value \\
\hline Age $(S D)$, years & $51.60(13.62)$ & $51.48(13.94)$ & 0.05 & 0.96 \\
\hline Gender: male, $n(\%)$ & $20(33.3)$ & $22(31.0)$ & 0.08 & 0.77 \\
\hline Length of medication, months (SD) & $112.40(123.24)$ & $129.17(117.35)$ & -0.74 & 0.46 \\
\hline \multicolumn{5}{|l|}{ Comorbidity, n (\%) } \\
\hline Diabetes & $22(49.2)$ & $20(75.4)$ & 9.53 & $0.02^{3}$ \\
\hline High blood pressure & $24(54.1)$ & $17(64.2)$ & 1.35 & 0.16 \\
\hline Asthma & $10(22.4)$ & $13(49.2)$ & 9.37 & $0.002^{\mathrm{a}}$ \\
\hline Depression & $21(46.6)$ & $13(46.0)$ & 0.003 & 0.55 \\
\hline $\begin{array}{l}\text { Chronic obstructive pulmonary } \\
\text { disease }\end{array}$ & $5(12.3)$ & $4(14.3)$ & 0.27 & 0.48 \\
\hline Cancer & $3(7.0)$ & $3(9.7)$ & 0.27 & 0.43 \\
\hline
\end{tabular}

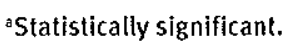


Table 2 Comparison of singing intervention vs. control in mean change in singing related QoL measures from baseline to 12 months.

\begin{tabular}{|c|c|c|c|c|c|c|c|}
\hline \multirow[b]{2}{*}{ Variables } & \multirow[b]{2}{*}{ Baseline (SD) } & \multicolumn{2}{|c|}{ Singing group } & \multicolumn{3}{|c|}{ Control group } & \multirow[b]{2}{*}{$p \cdot$ Value } \\
\hline & & Mean change (SE) & p-Value ${ }^{z}$ & Baseline (SD) & Mean change (SD) & $\mathrm{p}$-Value ${ }^{\mathrm{a}}$ & \\
\hline 1. Singing-related QoL & $10.33(8.50)$ & $-6.70(1.33)$ & $0.001^{\mathrm{c}}$ & $2.57(5.47)$ & $1.29(1.57)$ & 0.41 & $0.001^{\circ}$ \\
\hline 2. Singing-related positive affect & $23.96(18.87)$ & $-14.75(2.96)$ & $0.001^{\mathrm{c}}$ & $6.04(12.70)$ & $3.03(3.65)$ & 0.41 & $0.001^{c}$ \\
\hline 3. Resilience & $3.28(1.52)$ & $-0.20(0.27)$ & 0.47 & $2.97(2.16)$ & $-0.84(0.43)$ & 0.56 & 0.42 \\
\hline 4. Social isolation & $27.90(17.78)$ & $4.16(4.41)$ & 0.35 & $25.13(13.04)$ & $2.88(3.49)$ & 0.41 & 0.65 \\
\hline 5. Loneliness & $20.66(9.05)$ & $0.32(3.32)$ & 0.93 & $24.88(11.71)$ & $0.06(3.49)$ & 0.99 & 0.26 \\
\hline 6. Stress total score & $2.45(2.64)$ & $0.48(0.47)$ & 0.32 & $2.97(2.16)$ & $-0.31(0.74)$ & 0.67 & $0.11^{d}$ \\
\hline 7. Alcohol and jail term & $0.44(0.94)$ & $0.21(0.15)$ & 0.16 & $0.38(0.83)$ & $-0.42(0.27)$ & 0.13 & $0.02^{c}$ \\
\hline 8. Abuse accidents & $0.44(0.78)$ & $-.06(0.15)$ & 0.67 & $0.67(1.00)$ & $-0.02(0.29)$ & 0.95 & 0.46 \\
\hline 9. Job loss & $0.77(1.08)$ & $0.23(0.18)$ & 0.20 & $0.75(0.86)$ & $0.38(0.23)$ & 0.11 & 0.39 \\
\hline 10. Death of family & $0.65(0.68)$ & $0.05(0.12)$ & 0.66 & $0.89(0.69)$ & $0.01(0.18)$ & 0.95 & $0.08^{3}$ \\
\hline 11. Depression & $5.19(3.09)$ & $0.85(0.57)$ & 0.14 & $5.40(3.27)$ & $0.11(0.98)$ & 0.91 & 0.23 \\
\hline
\end{tabular}

${ }^{2}$ Comparison of within-group pre-post change. ${ }^{\circ}$ Comparison of mean change between singing and control group in postintervention time.

'Statistically significant. dMarginally statistically significant,

drug use and criminal events in the intervention group. There were marginally statistically significant reductions in the overall stress score and death of family member events in the singing group, as compared with the control group.

\section{Relationship between singing and social and emotional well-being}

Over the intervention period, significant correlations were demonstrated between singing-related QoL and social isolation, loneliness, total stress score, alcohol- and drugrelated problems, job loss, and unemployment-related stresses in the intervention group participants (Table 3). An increased level of QoL was associated with decreasing social isolation and loneliness and a low occurrence of stressful events including alcohol and drug use and criminal events.
Similarly, it can be seen in Table 4 that an increased level of positive affect through singing was associated with decreasing social isolation $(\mathrm{p}=0.001)$. No significant associations were seen between singing-related QoL and loneliness, stress, depression, and resilience.

\section{Discussion}

The key finding of our study is that a 12-month community singing program improves QoL and that this improvement was found in participants with chronic disease already on medical management. Furthermore, a relationship was found between improved QoL and social and emotional well-being as assessed by stress, social isolation, and loneliness. In the control group, there was no improvement in QoL and scores on stress-related events, social isolation, and loneliness actually increased over the study period. There was a trend toward reduced level of stress,

Table 3 Effects of singing-related QoL on stress, social and emotional well-being, resilience, social isolation, and toneliness in postintervention time in the intervention group.

\begin{tabular}{|c|c|c|c|c|}
\hline Varlables & B $(95 \%$ confldence interval) & p-Value & ANOVA & $\mathbf{R}^{2}, \%$ \\
\hline 1. Stress total score & $-0.48(-0.86$ to -0.09$)$ & $0.02^{\mathrm{a}}$ & 2.83 & 26.1 \\
\hline 2. Alcohol and jail term & $-0.10(-0.22$ to 0.02$)$ & $0.09^{\circ}$ & 5.04 & 38.7 \\
\hline 3. Abuse accidents & $-0.12(-0.27$ to 0.04$)$ & 0.14 & 0.96 & 11.2 \\
\hline 4. Job loss & $-0.19(-0.31$ to -0.07$)$ & $0.003^{3}$ & 4.70 & 38.0 \\
\hline 5. Death of family member & $-0.03(-0.14$ to 0.08$)$ & 0.59 & 0.96 & 10.7 \\
\hline 6. Social and emotional well-being & $-0.07(-0.59$ to 0.45$)$ & 0.78 & 0.09 & 1.4 \\
\hline 7. Resilience & $0.14(-0.07$ to 0.35$)$ & 0.19 & 1.80 & 18.4 \\
\hline 8. Social isolation & $-1.38(-3.46$ to 0.71$)$ & $0.08^{b}$ & 2.90 & 31.4 \\
\hline 9. Loneliness & $-2.38(-4.09$ to -0.66$)$ & $0.01^{\mathrm{a}}$ & 3.30 & 32.0 \\
\hline
\end{tabular}

Age and income are controlled in the models; age and alcohol-related problems, $B=-0.51(-0.88$ to 0.13$), p<0.01$.

atatistically significant. 'Marginally statistically significant. 
Table 4 Effects of singing-related relaxation on stress, social and emotional well-being, resilience, social isolation, and toneliness in postintervention time in the intervention group.

\begin{tabular}{|c|c|c|c|c|}
\hline Variables & B $(95 \%$ confldence interval) & p-Value & ANOVA & $R^{2}, \%$ \\
\hline 1. Stress total score & $-0.03(-0.08$ to 0.03$)$ & 0.30 & 2.20 & 5.8 \\
\hline 2. Alcohol and jail term & $-0.004(-0.02$ to 0.02$)$ & 0.65 & 2.16 & 5.9 \\
\hline 3. Abuse accidents & $0.002(-0.02$ to 0.02$)$ & 0.85 & 2.46 & 6.6 \\
\hline 4. Job loss & $-0.01(-0.03$ to 0.01$)$ & 0.42 & 0.49 & 1.4 \\
\hline 5. Death of family & $00.01(-0.03$ to 0.006$)$ & 0.19 & 0.59 & 1.6 \\
\hline 6. Depression & $-0.08(-0.15$ to 0.004$)$ & 0.04 & 5.48 & 14.6 \\
\hline 7. Resilience & $0.03(-0.01$ to 0.06$)$ & 0.17 & 4.15 & 10.9 \\
\hline 8. Social isolation & $0.84(0.27$ to 1.41$)$ & $0.01^{\mathrm{a}}$ & 3.11 & 9 \\
\hline 9. Loneliness & $0.03(-0.26$ to 0.32$)$ & 0.85 & 0.48 & 2.3 \\
\hline
\end{tabular}

Age and income are controlled in the models; age with resilience, $B=0.35(0.11$ to 0.58$), p<0.01 ;$ age and depression, $B=-0.74(-1.25$ to $-0.23), p<0.01$; age and altohol problems, $B=-0.16(-0.30$ to -0.03$), p<0.05$; age and abuse: $B=-0.20(-0.35$ to 0.05$), p<0.01$.

social isolation, and loneliness in the singing group, suggesting a possible improvement in social and emotional well-being in this group. Although no changes in depression and resilience were found, it should be noted that a decreased power to detect the effect of the singing program on improvement occurred due to sample size issues. For example, in the original cohort of 62 patients, we reported a significant improvement in depression and resilience in the singing vs. control group $(p=0.03)$. With the subset of 45 patients in this analysis, in the 12 months after intervention time, trends toward improvement in depression and resilience still exist $(p=0.08)$; however, the effect dwindles.

An increase in QoL through a singing program may be a robust marker of overall improved QoL in patients with multiple chronic disease. For these patients, poor physical and psychological health may cause excessive stress through circulation, respiratory, and non-respiratory mechanisms and may be associated with cardiovascular complications and airway inflammation [18, 19]. In contrast, positive physical and psychological health is associated with stable respiration and may protect people from triggered cardiac and respiratory events, thus demonstrating its potential restorative effects on cardiopulmonary and immune function. Improvements in QoL may therefore promote acute beneficial changes in hemodynamic indices as well as chronic changes in the overall hemodynamic profile. This study suggests that singing may be a potential adjunctive therapeutic option for patients with multiple chronic conditions that can improve QoL. Of significance, improved QoL for Aboriginal and Torres Strait Islander people with chronic conditions, as reflected by physical, psychological health, and social functioning, has not been previously described with singing activities.
The underlying mechanisms of improvement in QoL associated with singing in Aboriginal and Torres Strait Islanders may be speculated with reference to the extant literature. For example, singing has also been shown to affect the neurohormonal axis, which could contribute to an improved psychological state. Increased physical activity associated with singing has also been shown to improve immunity level [20]. In addition, the therapeutic practice inherent in singing may also contribute to increased phys. ical and psychological health. Singing training may facilitate increased respiratory muscle strength and control, and this has been observed in patients with quadriplegia [21]. These improvements in respiratory control could lead to improvements in breathing. Furthermore, singing includes a component of respiratory rhythm training with slow, deep, diaphragmatic breathing, which could play a role in retraining breathing patterns. Finally, the singing program may have indirectly promoted better sleep, improved mental health (e.g., decreased anxiety/ depression), and provided a social interaction opportunity for participants, which each may have contributed to improved QoL. Of particular note, we also observed a possible deterioration of mental health as seen in increased level of stress and depression in the control group. Possible explanations include natural progression of disease, disordered breathing, and group variability in QoL.

There is an association between QoL and social and emotional well-being, with physical and psychological health inversely correlating with decreasing levels of alcohol and drug use, criminal behavior, social isolation, and loneliness in our study. Interestingly, poor QoL is seen in several chronic disease states, including depression, diabetes, hypertension, and stroke $[1,2,5,9]$. This perception of poor QoL is typically associated with a poor social and emotional well-being in baseline study. The 
preliminary findings here suggest that singing-related improvements in patients may in part be mediated by effects on physical and psychological health.

There are several limitations of this study. First, a clinical evaluation of the patients was not undertaken. Thus, changes in real physical health in the study subjects are not known. In addition, the sample size of patients with cardiac disease is small, and it is likely that there may have been insufficient power to detect relationships between changes in QoL measures and depression and resilience measures. We also cannot determine what component of singing is responsible for the observed benefits. Physical activity may have important effects, and it is unclear what added role breathing and relaxation may play. Finally, we have no data on direct physiological effects during singing, so that possible mechanistic effects remain speculative. Despite these limitations, the data presented provide valuable preliminary information for generating testable hypotheses on the potential effects of QoL of group singing in patients with chronic disease.

In conclusion, our preliminary findings suggesting that a 12-month singing program may improve QoL in patients with multiple chronic diseases on various medications. As QoL has the potential to improve the cardiorespiratory profile in chronic disease and have potential protective effects, a further evaluation of this approach is needed. Further research should include physiological testing to document function, active controls (e.g., conventional exercise) to better understand component effects of therapeutic singing, and outcomes directly assessing physical function, hemodynamics, and respiratory changes during singing. In addition, a more detailed examination of the study popula. tion, in areas such as multiple chronic diseases associated with and without cerebrovascular/neurologic (stroke) and psychiatric (alcohol, anxiety, and depression) conditions may provide insights into the mechanisms of the effects of singing on QoL and social and emotional well-being.

Acknowledgments: The authors received financial support from Griffith Health Institute of Griffith University, Queensland Aboriginal and Islander Health Council in Australia, and Queensland Centre for Social Science Innovation in Queensland, Australia. The authors also wish to acknowledge the support of the following Abo. riginal Community Controlled Health Services: Kambu Medical Services Inc., Kalwun Health Service, Brisbane Aboriginal and Torres Strait Islander Community Health Service Brisbane Ltd., Goolburri Health Advancement Corporation, and Warwick Community Peace Festival. The authors also wish to thank all the participants from five Aboriginal and Torres Strait Islander communities. The authors also want to thank Lindsay Johnson's assistance with data collection and work.

\section{Conflict of interest statement}

Authors' conflict of interest disclosure: Authors declare there are no conflict of interests.

Received June 24, 2012; accepted September 2, 2012; previously published online October 12, 2012

\section{References}

1. Lam CL, tauder I). The impact of chronic diseases on the healthrelated quality of life (HRQOL) of Chinese patients in primary care. Fam Pract 2000;17:159-69.

2. Thommasen HV, Zhang W. Impact of chronic disease on quality of life in the Bella Coola Valley. Rural Remote Health 2006;6:1-18.

3. Australian Institute of Health and Welfare. Measuring the social and emotional wellbeing of Aboriginal and Torres Strait Islander peoples. AlHW Cat. No. IHW 24. Canberra, Australia: AlHW, 2009:115.

4. Australlan Institute of Health and Welfare and Australian Bureau of Statistics. The health and welfare of Australia's Aboriginal and Torres Strait Islander peoples. ABS Cat. No. 4704.0 Canberra, Australia: AlHW/ABS, 2008.

5. Walker AE. Multiple chronic diseases and quality of life: patterns emerging from a large national sample, Australia. Chronic Illn 2007;3:202-18.

6. Sprangersa MA, de Regla EB, Heleen AF, van Agtc ME, Bijld RV, de Boere JB, et al. Which chronic conditions are associated with better or poorer quality of life? I Clin Epidemiol 2000;53: 895-907.
7. Mapes DL, Lopes AA, Satayathum S, Mccullough KP, Goodkin $D A$, Locatelli $F$, et al. Health-related quality of life as a predictor of mortality and hospitalization: the Dialysis Outcomes and Practice Patterns Study. Kidney Int 2003;64:339-49.

8. Iqbal ), Francis L, Reid J, Murray S, Denvir M. Quality of life in patients with chronic heart failure and their carers: a 3.year follow-up study assessing hospitalization and mortality. Eur I Heart Fail 2010;12:1002-8.

9. Appels A, van Elderen T, Bar F. Effects of a behavioural intervention on quality of life and related variables in angloplasty patients: results of the EXhaustion Intervention Trial. I Psychosom Res 2006;61:1-7; discussion 9-10.

10. Clift S, Morrison I. Group singing fosters mental health and wellbeing: findings from the East Kent "singing for health" network project. Mental Health Soc Incl 2011;15: 88-97.

11. Riley $\mathrm{KL}$, Gridley $\mathrm{H}$. Harmony in the community: group perspectives on the health benefits of singing. UNESCO Observatory, Faculty of Architecture, Building and Planning, The University of Melbourne Referred E.Journal 2010;2:1-18. 
12. Sun J, Buys N. Eflectiveness of participatory community singing and mental health promotion in Aboriginal and Torres Strait Islander people. In: Victor 0 , editor. Essential notes in psychiatry [online publication]. InTech; 2012.

13. Clift $S$, Hancox G, Staricoff $R$, Whitmore C. A systematic mapping and review of research on singing and health-non-clinical studies, Canterbury: Canterbury Christ Church University College, 2008.

14. Lob GV, Camic $P$, Clift $S$. The use of singing in a group as a response to adverse life events. Int I Mental Health Promotion 2011;12:45-53.

15. National Heart Foundation of Australia. Secondary prevention of cardiovascular disease, 2010.

16. Kowal E, Gunthorpe W, Bailie RS. Measuring emotional and social wellbeing in Aboriginal and Torres Strait Islander populations: an analysis of a negative life events scale. Int J Equily Health 2007;6:1-12.
17. Kessler RC, Andrews G, Colpe LJ, Hiripi E, Mroczek DK, Normand $\mathrm{SL}$, et al. Short screening scales to monitor population prevalences and trends in non-specific psychological distress. Psychol Med 2002;32:959-6.

18. Schlesinger CM, Ober C, McCarthy MM, Watson JD, Seinen A. The development and validation of the Indigenous Risk Impact Screen (IRIS): a 13.item screening instrument for alcohol and drug and mental health risk. Drug Alcohol Rev 2007;26:109-17.

19. Lee RM, Draper M, Lee S. Social connectedness, dysfunctional interpersonal behaviors, and psychological distress: testing a mediator model. J Couns Psychol 2001:310-8.

20. Abelha Ff, Botelho $M$, Fernandes V, Barros $H$. Quality of life and mortality assessment in patients with major cardiac events in the postoperative period. Rev Bras Anestesiol 2010;60:268-84.

21. Wright RJ, Rodriguez M, Cohen S. Review of psychosocial stress and asthma: an integrated biopsychosocial approach. Thorax 1998;53:1066-74. 